\title{
AN ADDITION THEOREM FOR SETS OF ELEMENTS OF ABELIAN GROUPS
}

H. B. MANN

Let $G$ be a finite Abelian group, $A, B$ sets of elements in $G$. By $A B$ we denote the set of all elements of the form $a b(a \in A, b \in B)$, by $\bar{A}$ the complement of $A$ in $G$, and by $(A)$ the number of elements in $A$. We shall prove the following theorem.

THEOREM. If for the set $A$ and every subgroup $H$ of $G$

$$
(A H) \geqq(A)+(H)-1 \text { or } A B=G,
$$

then for every set $B$

$$
(A B) \geqq(A)+(B)-1 \text { or } A B=G .
$$

For the proof we need the following two theorems proved in [1].

(i) If $A, B$ are sets of elements of a finite group $G$, then

$$
(G) \geqq(A)+(B) \text { or } G=A B .
$$

(ii) If $G$ is a finite Abelian group $c \in G, c \notin A B$, then there exists a set $B^{*} C G$ such that

(1) $B^{*} \supseteq B$

(2) $\overline{A B^{*}}=c H$, where $H$ is a sub-group of $G$,

(3) $\left(A B^{*}\right)-(A B)=\left(B^{*}\right)-(B)$.

Proof of the TheOREM. By virtue of (ii) it is sufficient to prove the theorem for the case that $\overline{A B}=c H$ where $H$ is a subgroup of $G$. Consider then the factor group $G / H$ and let $A^{\prime}, B^{\prime}$ be the set of cosets of $H$ contained in $A H, B H$ respectively. Then by (i)

$$
(G / H) \geqq\left(A^{\prime}\right)+\left(B^{\prime}\right)
$$

But $(G / H)(H)=(G), \quad\left(B^{\prime}\right)(H) \geqq(B)$, and by hypothesis $\left(A^{\prime}\right)(H)$ $=(A H) \geqq(A)+(H)-1$. Multiplying $(4)$ by $(H)$ we therefore get

$$
(G) \geqq(A)+(B)+(B)-1
$$

and

$$
\begin{gathered}
(A B)=(G)-(H) \geqq(A)+(B)-1 . \\
\text { REFERENCE }
\end{gathered}
$$

1. H. B. Mann, On products of sets of group elements, Canadian Journal of Mathematics vol. 4 (1952) pp. 64-66.

The OHo State University

Received by the editors August 11, 1952. 